\title{
On admissible hybrid Geraghty contractions
}

\section{ERdal Karapinar ${ }^{1,2}$, Adrian Petruşel ${ }^{3,4}$ and Gabriela PetruşeL ${ }^{5}$}

\section{ABSTRACT.}

In this manuscript, we introduce the notion of admissible hybrid Geraghty contraction and we investigate the existence of fixed points of such mappings in the setting of complete metric spaces. Our results not only extend and generalize several results in the fixed point theory literature, but also unify most of them. We give some corollaries to illustrate the novelty of the main result.

\section{REFERENCES}

[1] Agarwal, R. and Karapınar, E., Interpolative Rus-Reich-Ciric type contractions via simulation functions, An. St. Univ. Ovidius Constanta, Ser. Mat., 27 (2019), No. 3, 137-152

[2] Aydi, H., Chen, C. M. and Karapınar, E., Interpolative Ćirić-Reich-Rus type contractions via the Branciari distance, Mathematics, 7 (2019), No. 1, 84; https://doi.org/10.3390/math7010084

[3] Aydi, H., Karapınar, E. and Roldan Lopez de Hierro, A. F., w-Interpolative Ćirić-Reich-Rus-type contractions, Mathematics 7 (2019), No. 1, 84; https://doi.org/10.3390/math7010084

[4] Bianchini, R. M. and Grandolfi, M. , Transformazioni di tipo contracttivo generalizzato in uno spazio metrico, Atti Acad. Naz. Lincei, VII. Ser. Rend. Cl. Sci. Fis. Mat. Natur., 45 (1968), 212-216

[5] Ćirić, Lj. , A generalization of Banach's contraction principle, Proc. Amer. Math. Soc., 45 (1974), 267-273

[6] Dhage, B. C., Hybrid fixed point theory in partially ordered normed linear spaces and applications to fractional integral equations, Diff. Equations and Appl., 5 (2013), 155-184

[7] Geraghty, M., On contractive mappings, Proc. Amer. Math. Soc., 40 (1973), 604-608

[8] Hardy, G. E. and Rogers, T. D., A generalization of a fixed point theorem of Reich, Canad. Math. Bull., 16 (1973), 201-206

[9] Kannan, R, Some results on fixed points, Bull. Calcutta Math. Soc., 60 (1968), 71-76

[10] Karapınar, E., Alqahtani, O. and Aydi, H., On interpolative Hardy-Rogers type contractions, Symmetry, 11 (2019), No. 1, 8; https://doi.org/10.3390/sym11010008

[11] Karapinar, E., Revisiting the Kannan type contractions via interpolation, Adv. Theory Nonlinear Anal. Appl., 2 (2018), 85-87

[12] Karapınar, E., Agarwal, R. and Aydi, H., Interpolative Reich-Rus-Ćirić type contractions on partial metric spaces, Mathematics, 6 (2018), No. 11, 256; https://doi.org/10.3390/math6110256

[13] O'Regan D. and Petruşel, A., Fixed point theorems for generalized contractions in ordered metric spaces, J. Math. Anal. Appl., 341 (2008), 1241-1252

[14] Petruşel, A. and Rus, I. A., Fixed point theorems in ordered L-spaces, Proc. Amer. Math. Soc., 134 (2006), 411-418

[15] Petruşel, A. and Rus, I. A., Fixed point theory in terms of a metric and of an order relation, Fixed Point Theory, 20 (2019), 601-622

[16] Popescu, O., Some new fixed point theorems for $\alpha$-Geraghty contractive type maps in metric spaces, Fixed Point Theory Appl., 2014, 2014:190

Received: 05.01.2020; In revised form: 12.05.2020; Accepted: 19.05.2020

2010 Mathematics Subject Classification. 47H10, 54H25, 46J10.

Key words and phrases. Hybrid contraction, Geraghty type contraction, Fixed point theory, metric space.

Corresponding author: Erdal Karapinar; karapinar@mail.cmuh.org.tw 
[17] Radenovic, S., Kadelburg, Z., Jandrlic, D. and Jandrlic, A., Some results on weakly contractive maps, Bull. Iranian Math. Soc., 38 (2012), 625-645

[18] Reich, S., Some remarks concerning contraction mappings, Canad. Math. Bull., 14 (1971), 121-124

[19] Rus, I. A., Generalized Contractions and Applications, Cluj University Press, Clui-Napoca, Romania, 2001

[20] Rus, I. A., Petruşel, A. and Petruşel, G., Fixed Point Theory, Cluj University Press, Cluj-Napoca, 2008

[21] Samet, B., Vetro, C. and Vetro, P., Fixed point theorems for $\alpha-\psi$-contractive type mappings, Nonlinear Anal. TMA, 75 (2012), 2154-2165

[22] Watanabe, T., Fixed point theorems in ordered metric spaces and applications to nonlinear boundary value problems, Fixed Point Theory, 20 (2019), 349-364

${ }^{1}$ Department of Medical Research

China Medical University

China Medical University Hospital

40402, TAICHUNG, TAIWAN

${ }^{2}$ ÇANKAYA UNIVERSITY

DEPARTMENT OF MATHEMATICS

06790, ETIMESGUt, ANKARA, TURKEy

Email address: erdalkarapinar@yahoo.com

${ }^{3}$ Department of Mathematics

BABEŞ-BOLYAI UNIVERSITY

CLUJ-NAPOCA, ROMANIA

${ }^{4}$ ACADEMY OF ROMANIAN SCIENTISTS, BUCHAREST, ROMANIA

Email address: petrusel@math.ubbcluj.ro

${ }^{5}$ DePARTMENT OF Business BABEŞ-BOLyai UNIVERSity

Cluj-Napoca, Romania

Email address: gabi.petruseletbs.ubbcluj.ro 